\title{
Nosocomial Acinetobacter Infections in Intensive Care Unit
}

\author{
${ }^{1}$ Nwadike V. Ugochukwu, ${ }^{2}$ Fayemiwo S. Adetona, \\ ${ }^{2}$ Fowotade Adeola, ${ }^{2}$ Bakare R. Ajani and ${ }^{2} \mathrm{O}$. Olusanya \\ ${ }^{1}$ Department of Medical Microbiology and Parasitology, Federal Medical Center, Owerri, Nigeria \\ ${ }^{2}$ Department of Medical Microbiologyand Parasitology, College of Medicine, University of Ibadan, Nigeria
}

Received 2012-09-08, Revised 2013-06-07; Accepted 2013-06-08

\begin{abstract}
Acinetobacter plays an important role in the infection of patients admitted to hospitals. Acinetobacter are free living gram-negative coccobacilli that emerge as significant nosocomial pathogens in the hospital setting and are responsible for intermittent outbreaks in the Intensive Care Unit. The aim of this study was to determine the prevalence of Acinetobacter in patients admitted into the Intensive Care Unit and determine their role in infections in the ICU. A total of one hundred patients were recruited for the study, catheter specimen urine, tracheal aspirate and blood culture were collected aseptically from the patients. The specimens were cultured on blood and MacConkey and the organisms identified using Microbact 12E (0xoid). The Plasmid analysis was done using the TENS miniprep method. Fourteen (14\%) of the 100 patients recruited into the study, developed Acinetobacter infection. Acinetobacter spp constituted $9 \%$ of the total number of isolates. Twelve $(86 \%)$ of the isolates were recovered from tracheal aspirate, $1(7 \%)$ from urine and $1(7 \%)$ from blood. All of the isolates harbor plasmids of varying molecular sizes. Ten of the fourteen Acinetobacter were isolated at about the same period of time in the ICU with $6(42.7 \%)$ having plasmid size in the $23.1 \mathrm{~kb}$ band and all showed similar pattern revealing that the isolates exhibit some relatedness. The clonal nature of the isolates suggest that strict infection control practices must be adopted in ICU, also an antibiotic policy must be developed for the ICU to prevent abuse of antibiotics that may lead to selection of resistant bacteria.
\end{abstract}

Keywords: Acinetobacter, Intensive Care Unit

\section{INTRODUCTION}

The control of hospital-acquired infection caused by multidrug resistant Gram-negative bacilli has proved to be a peculiar problem. In the 1970 s an increase in the resistant members of the family Enterobacteriaecea involved in nosocomial infections followed the introduction of newer broad spectrum antibiotics in hospitals and this led to an increase in the importance of aerobic Gram-negative bacilli, including Pseudomonas aeruginosa and Acinetobacter spp (Gerner-Smidt, 1994).

Acinetobacter is ubiquitous, free-living and fairly stable in the environment (Joshi et al., 2006). Due to their distinct adhesive ability to epithelial cells; they have a predilection to colonize skin, especially in the areas of the perineum, inguinal region, axillae, mucous membranes and upper respiratory airway and cause human infections which include pneumonia, septicemia, wound sepsis, urinary tract infection, endocarditis and meningitis (Joshi et al., 2006; GarciaGarmendia et al., 2001a).

Acinetobacter infections are increasingly implicated in infections in intensive care units and have been cited in up to $17 \%$ of Ventilator Associated Pneumonia, second only to Pseudomonas, which was responsible for $19 \%$ of Ventilator Associated Pneumonia in an ICU (Munoz-Price and Weinstein, 2008). In a review from the CDC, $7 \%$ of ICU-acquired pneumonias were due to

Corresponding Author: Nwadike V. Ugochukwu, Department of Medical Microbiology and Parasitology, Federal Medical Center, Owerri, Nigeria 
Acinetobacter in 2003 compared to $4 \%$ in 1986 (Gaynes et al., 2005).

Infections caused by Acinetobacter are difficult to control due to multi-drug resistance, this limits therapeutic options in critically ill and debilitated patients especially from intensive care units where their prevalence is most noted (Joshi et al., 2006).

Acinetobacter outbreaks have been traced to contamination of respiratory-therapy and ventilator equipment from cross-infection by the hands of health care workers who have cared for colonized, infected patients or touched contaminated fomites (Villegas and Hartstein, 2003; Maragakis et al., 2004).

A. baumannii, A. calcoaceticus and A. lwoffii are the Acinetobacter species most frequently reported in clinical literature (Garcia-Garmendia et al., 2001b). There is some data to suggest that the proportion of Intensive Care Unit (ICU)-acquired pneumonia cases being found to be due to $A$. baumannii is on the increase.

In large surveillance studies from the United States between 5 and $10 \%$ of cases of ICU-acquired pneumonia were due to A. baumannii. Data on Acinetobacter in Africa is largely limited to South Africa at the present time, although there are scattered reports from other countries in Africa (Lowman et al., 2008).

Prior to this report there has been no published data on Acinetobacter infection in the ICU of University College hospital Ibadan. Acinetobacter infection in literature is associated with high morbidity, mortality and increased length of hospital stay especially amongst patients in ICU, this has been attributed to its ability to acquire and up regulate resistance genes (Garcia-Garmendia et al., 2001a).

The aim of the study was to determine the prevalence of Acinetobacter in the intensive care unit and also to determine the relatedness of the isolates

\section{MATERIALS AND METHODS}

This cross sectional study was carried out in the University College 'Hospital (UCH) Ibadan, Nigeria. A total of one hundred patients were recruited into study from the ICU which is 12 bedded and has a monthly turnover of 25 patients. This population of patients comprised of patients who have had surgery and are on ventilators or intubation with a prior history of antibiotic use and those who have had any form of instrumentation.
Ethical approval was obtained from the University of Ibadan/University College Hospital joint Ethical Committee.

A written informed consent was also obtained from guardians, spouse, parent or caregiver of each participant, thereafter relevant medical history, sociodemograhic data and other information obtained from the care giver and case files were entered into a study proforma.

\subsection{Specimen Collection and Transport}

Tracheal aspirate, blood and catheter specimen urine were collected from all recruited patients for microscopy, culture and sensitivity. Specimens were collected using aseptic technique to prevent contamination.

For optimal results, specimens were collected in clean sterile, wide bore containers. The samples were collected in patients who had spent at least $48 \mathrm{~h}$ in ICU.

\subsection{Microscopy}

A Gram stain was done on smears made from specimens and then viewed under the light microscope at x100. Classically Acinetobacter spp. appear as; short, plump, Gram-negative rods.

\subsection{Culture and Identification}

The specimen was inoculated on MacConkey agar and blood agar and incubated at $35-37^{\circ} \mathrm{C}$ for $18-24 \mathrm{~h}$. Acinetobacter species grew on MacConkey agar appearing as non lactose fermenters.

All Gram-negative coccobacilli isolated were tested for catalase and motility. All catalase positive, non-motile Gram negative coccobacilli were subjected to an oxidase test. All oxidase negative organisms were inoculated into peptone broth for about $30 \mathrm{~min}$. Subsequently $1 \mathrm{ml}$ of the broth was inoculated into the various cups of Microbact Identification kit (Oxoid) and incubated for 18-24 h. After the stated period, Gram negative coccobacilli were identified as Acinetobacter spp based on the reactions on the identification panel which was read with the help of the identification software that accompanied the kit.

\subsection{Plasmid Analysis}

The plasmid analysis was done in collaboration with the molecular biology laboratory of the Nigerian Institute of Medical Research Yaba Lagos, Nigeria, using the 
TENS method. This was done to determine the relatedness of the isolates.

\subsection{Plasmid Extraction}

About $1.5 \mathrm{~mL}$ of overnight culture was centrifuged at $10,000 \mathrm{rpm}$ for $1 \mathrm{~min}$ in a micro-centrifuge to pellet cells. The supernatant was gently decanted leaving $100 \mu \mathrm{L}$ together with cell pellet; the resulting suspension was vortexed at high speed to re suspend cells completely.

A volume of $300 \mu \mathrm{L}$ of TENS was added to the mixture and mixed until the mixture becomes sticky, 150 $\mu \mathrm{L}$ of $3.0 \mathrm{M}$ sodium acetate $\mathrm{pH} 5.2$ was added to the mixture and vortexed to mix completely.

The mixture was spun for $5 \mathrm{~min}$ in a micro-centrifuge to pellet chromosomal DNA and supernatant was discarded, the pellet was rinsed twice with $1 \mathrm{~mL}$ of $70 \%$ ethanol and the pellet re-suspended in $40 \mu$ l of distilled water for further use:

$$
\left(\begin{array}{l}
\text { TENS composition: Tris } 25 \mathrm{mM} \text {, } \\
\text { EDTA } 10 \mathrm{mM}, \mathrm{NaOH} 0.1 \mathrm{~N} \text { and SDS } 0.5 \% \text {. }
\end{array}\right)
$$

\subsection{Agarose Gel Electrophoresis}

Agarose Gel Electrophoresis was the separation method used to separate DNA based on their molecular weight. A load of $0.8 \mathrm{~g}$ of agarose powder was added to $100 \mathrm{mls}$ of TAE buffer and dissolved by boiling. It was allowed to cool to about $60^{\circ} \mathrm{C}$ then $10 \mathrm{uL}$ of ethidium bromide added and mixed by swirling gently. The agar was poured into electrophoresis tray with the comb in place to obtain a gel thickness of about $4-5 \mathrm{~mm}$. It was allowed to solidify then comb removed and the tray placed in the electrophoresis tank.

TAE buffer was poured into the tank ensuring that the buffer was covering the surface of the gel. A volume of $10 \mu \mathrm{L}$ of sample was mixed with $2 \mu \mathrm{L}$ of the loading dye and the samples were carefully loaded into the wells created by the combs. The marker was loaded on lane 1 followed by the samples. The electrodes were connected to the power pack in such a way that the negative terminal was at the end where the sample has been loaded. Electrophoresis was run at 60 volt until the loading dye had migrated about three-quarter of the gel. The electrodes were disconnected and turned off and the gel viewed on a UV-transilluminator.

TAE: (Tris, Acetic acid and EDTA).

\subsection{Data Analysis}

All data were analyzed using the Statistical Package for the Social Sciences (SPSS) version 15.0. Data were presented using frequency tables, charts, as appropriate and cross tabulation to study relationships and association between variables.

\section{RESULTS}

A total of one hundred patients were recruited into the study over a period of nine months. The age of the patients ranged from 2 years to 95 years. Majority of the patients $(40 \%)$ were in the 31-40year age group while the 10-20 year age group constituted the least age group (4\%). There were 52 males and 48 males, giving a male to female ratio of 1.08:1. Forty Eight (48\%) were Christians, forty seven (47\%) were Muslims and $5 \%$ were traditionalists. Eighty one ( $81 \%)$ of the patients were admitted from the Accident and Emergency unit while 19\% were from other wards in the hospital, Fifty eight (58\%) of the patients were resident in Ibadan while forty two $(42 \%)$ of the patients were from outside Ibadan (Table 1).

Table 1. Sociodemographic data of ICU patients

\begin{tabular}{lrr}
\hline Variable & Number & Percentage \\
\hline Age (years) & 13 & \\
$<10$ & 4 & 13.0 \\
$11-20$ & 13 & 4.0 \\
$21-30$ & 30 & 13.0 \\
$31-40$ & 20 & 30.0 \\
$41-50$ & 10 & 20.0 \\
$51-60$ & 10 & 10.0 \\
$>60$ & & 10.0 \\
Sex & 52 & \\
Male & 48 & 52.0 \\
Female & & 48.0 \\
Source of admission & 82 & \\
A/E & 18 & 82.0 \\
Ward & & 18.0 \\
Residence & 58 & \\
Ibadan & 42 & 58.0 \\
Outside Ibadan & & 42.0 \\
Religion & 48 & 48.0 \\
Christian & 47 & 47.0 \\
Islam & 5 & 5.0 \\
Traditionalists & & \\
\hline
\end{tabular}


Table 2. Frequency distribution of isolates from patient samples

\begin{tabular}{|c|c|c|}
\hline Organisms & Frequency & Percentage \\
\hline \multicolumn{3}{|l|}{ Tracheal Aspirate } \\
\hline Acinetobacter spp & 12 & 12 \\
\hline Candida albicans & 1 & 1 \\
\hline Citrobacter freundii & 1 & 1 \\
\hline Enterobacter spp & 1 & 1 \\
\hline Escherichia coli & 9 & 9 \\
\hline Escherichia vulneris & 1 & 1 \\
\hline Klebsiella pneumonia & 10 & 10 \\
\hline Klebsiella aerogenes & 7 & 7 \\
\hline Klebsiella oxytoca & 3 & 3 \\
\hline Proteus mirabilis & 3 & 3 \\
\hline Proteus vulgaris & 2 & 2 \\
\hline Pseudomonas aeruginosa & 10 & 10 \\
\hline Staphylococcus aureus & 11 & 11 \\
\hline Viridans Streptococci & 3 & 3 \\
\hline \multicolumn{3}{|l|}{ Urine } \\
\hline Acinetobacter spp & 1 & 1 \\
\hline Candida albicans & 4 & 4 \\
\hline Citrobacter freundii & 1 & 1 \\
\hline Enterococcus faecalis & 1 & 1 \\
\hline Escherichia coli & 8 & 8 \\
\hline Klebsiella ozaenae & 2 & 2 \\
\hline Klebsiella aerogenes & 1 & 1 \\
\hline Klebiella oxytoca & 6 & 6 \\
\hline Klebsiella pneumonia & 9 & 9 \\
\hline Proteus mirabilis & 1 & 1 \\
\hline Proteus vulgaris & 3 & 3 \\
\hline Pseudomonas aeruginosa & 2 & 2 \\
\hline Staphylococcus aureus & 6 & 6 \\
\hline \multicolumn{3}{|l|}{ Blood } \\
\hline Acinetobacter spp & 1 & 1 \\
\hline Candida albicans & 1 & 1 \\
\hline Citrobacter freudii & 1 & 1 \\
\hline Escherichia coli & 3 & 3 \\
\hline Klebsiella aerogenes & 3 & 3 \\
\hline Klebsiella oxytoca & 1 & 1 \\
\hline Klebsiella ozaenae & 3 & 3 \\
\hline Pseudomonas aeruginosa & 1 & 1 \\
\hline Staphylococcus aureus & 17 & 17 \\
\hline
\end{tabular}

Acinetobacter spp. was isolated from fourteen (14\%) of the total number of patients recruited into the study and was responsible for $14 \%$ of infections in the ICU based on evaluation of clinical charts. It represented $9 \%$ of the isolates from all the specimens collected during the study period (Table 2). Acinetobacter was responsible for $1 \%$ of urinary tract infections, $14 \%$ of respiratory tract infections and $1 \%$ of blood stream infections.

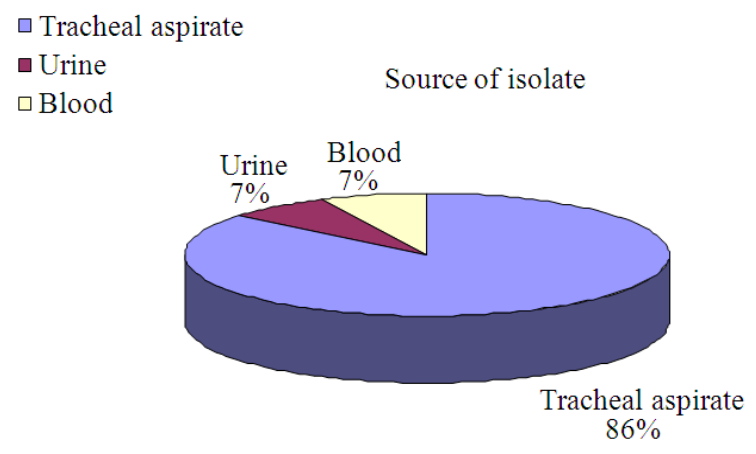

Fig. 1. Frequency distribution of isolates

Twelve $(86 \%)$ of the isolates were recovered from tracheal specimens while 1(7\%) was from urine and blood specimens each. Eleven (79\%) of the isolates were Acinetobacter baumannii while the others were Acinetobacter iwoffi (14\%) and Acinetobacter calcoaceticus (7\%), (Fig. 1).

All the isolates in the study harbor plasmids of different molecular sizes. Ten of the fourteen isolates were isolated at about the same period in the ICU with $6(42.7 \%)$ having plasmid size in the $23.1 \mathrm{~kb}$ band and having similar pattern showing that they may be related. The remaining $9(67.3 \%)$ did not show any appreciable bands that were related to the others (Fig. 2).

\section{DISCUSSION}

The prevalence of Acinetobacter infection in this study was $14 \%$, this high rate of Acinetobacter infection may be attributed to the poor infection control practices in the ICU of the Hospital. The observed prevalence is higher than reports from similar studies carried out in France by Joly-Guillou (2005) who reported 9\% and Iregbu et al. (2002) who reported $4.6 \%$ in Lagos, Nigeria (Iregbu et al., 2002). Acinetobacter constituted $9 \%$ of all isolates in the study, this finding is low compared to $14.5 \%$ obtained by Kessaris et al. (2006), $13.9 \%$ by Raka et al. (2004) but higher than $8.4 \%$ reported by Oberoi et al. (2009) and 3\% reported by Iregbu et al. (2002). This may be because their study included all patients in the hospital compared to this study which was limited to the ICU.

The incidence of Acinetobacter blood stream infection in this study was $1.3 \%$, this is a little lower than $2 \%$ reported by Michalopoulos and Falagas (2007) and $8.8 \%$ reported by Garcia-Garmendia et al. (2001b) This difference may be due to the lower number of patients recruited in the study. 


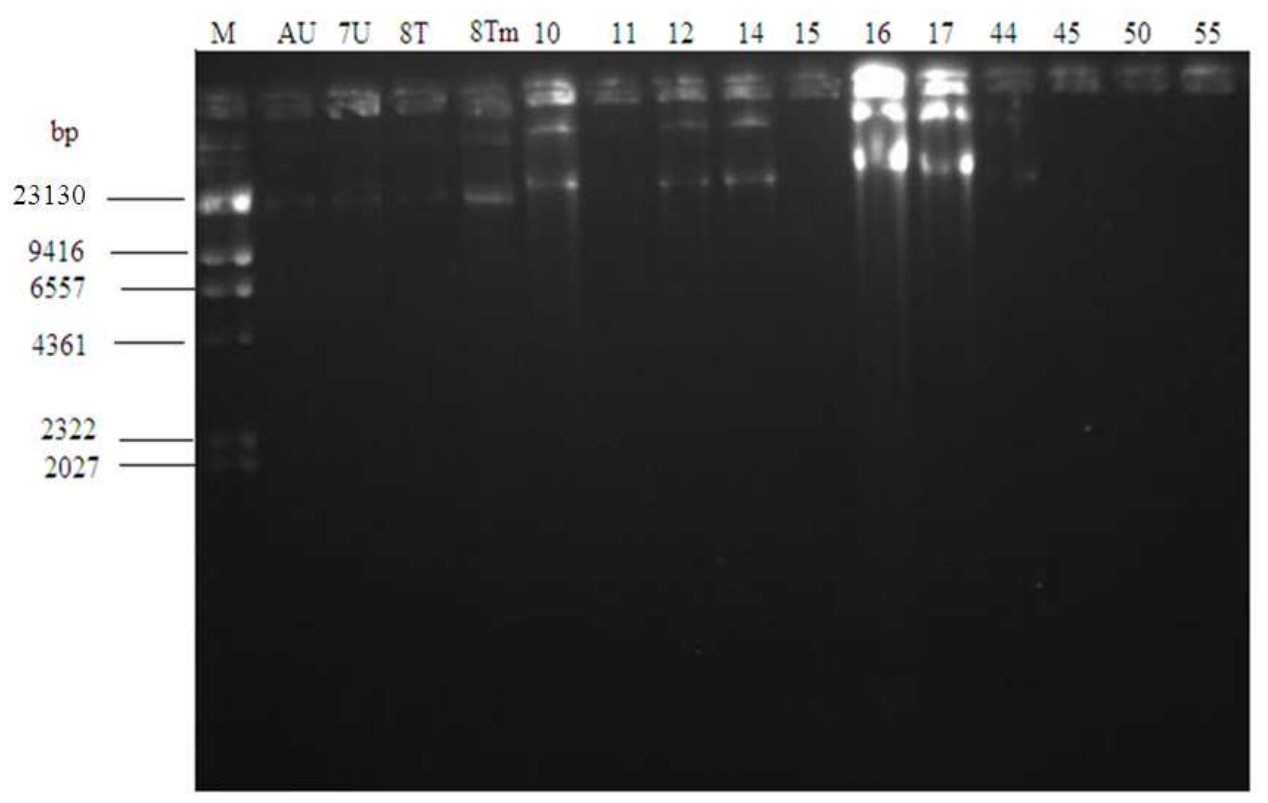

Fig. 2. Showing the plasmids of the Acinetobacter isolates M- Molecular ladder to serve as ruler for the procedure. The numbered and lettered wells represent different patient isolates. (AU, 7U, 8T, 10-55: Represent Acinetobacter isolates from different patients)

Prior to the introduction of current advanced resuscitatory devices, Acinetobacter spp were mainly recovered from the urinary tract however with the advent of mechanical ventilation most of the organisms are now being isolated from respiratory tract specimens. In this study, majority $12(86 \%)$ of the isolates were recovered from tracheal aspirate. This finding is similar to the $87 \%$ observed by Raka et al. (2004) but much higher than $46.5 \%$ reported by Popescu et al. (2011). The high rate of recovery from the respiratory tract may be due to the invasive procedures that are carried out in the respiratory tract in the process of maintaining the airway. The low recovery rate (1\%) of Acinetobacter spp from the urinary tract is similar to the $1.6 \%$ rate reported in the NNIS study (Gaynes et al., 2005). This further confirms the popular report that Acinetobacter is no longer a common uropathogen.

Severe underlying diseases, invasive diagnostic and therapeutic procedures used in ICUs have been demonstrated to predispose patients to severe infections with A. baumannii (Jarousha et al., 2008). In recent years, A. baumannii has become an important pathogen especially in intensive care units. Persistence of endemic A. baumannii isolates in ICU seems to be related to their ability for long-term survival on inanimate surfaces in patient's immediate environment and their widespread resistance to the major antimicrobial agents (Oberoi et al.,
2009). Our finding of A.baumannii as the major pathogen in this study is therefore not too surprising as this has been reported earlier by Joshi et al. (2006).

All the clinical isolates of Acinetobacter in the study harbor plasmids of different molecular sizes. Ten of the isolates were isolated at about the same period of time with $6(42.7 \%)$ having plasmid size in the $23.1 \mathrm{~kb}$ band and exhibit similar patterns showing that they may have originated from the same clone and a signal to potential outbreak strain. Outbreaks of Acinetobacter infections are linked to contaminated respiratory equipment, intravascular access devices, bedding materials and transmission via hands of hospital personnel. Acinetobacter outbreaks have been traced to commonsource contamination, particularly contaminated respiratory-therapy and ventilator equipment, crossinfection by the hands of health care workers who have cared for colonized or infected patients or touched contaminated formite and to the occasional health care worker who carries an epidemic strain (Raka et al., 2004).

\section{CONCLUSION}

In conclusion the ICU is responsible for providing life support services to patients from diverse specialties. The isolation of Acinetobacter among 
critically ill patients in the ICU is a cause for concern. There is an urgent need for education of health care workers in the ICU on proper infection control practices. There is also a need for active surveillance for Acinetobacter spp in the ICU.

\section{REFERENCES}

Garcia-Garmendia, J.L., C. Ortiz-Leyba, J. GarnachoMontero, F.J. Jimenez-Jimenez and C. PerezParedes et al., 2001a. Risk factors for Acinetobacter baumannii nosocomial bacteremia in critically ill patients: A cohort study. Clin. Infect. Dis., 33: 939946. DOI: $10.1086 / 322584$

Garcia-Garmendia, J.L., C. Ortiz-Leyba, J. GarnachoMontero, F.J. Jimenez-Jimenez and C. PerezParedes et al., 2001b. Risk factors for acinetobacter baumannii nosocomial bacteremia in critically Ill patients: A cohort study. Clin. Infect. Dis., 33: 939946. DOI: $10.1086 / 322584$

Gaynes, R.A., R. Gaynes and J.R. Edwards, 2005. Overview of nosocomial infections caused by GramNegative Bacilli. Clin. Infect. Dis., 41: 848-854. DOI: $10.1086 / 432803$

Gerner-Smidt, P., 1994. Acinetobacter: Epidemiological and taxonomic aspects, APMIS Suppl., 47: 1-41. PMID: 7811530

Iregbu, K.C., F.T. Ogunsola and T.O. Odugbemi, 2002. Infections caused by Acinetobacter species and their susceptibility to 14 antibiotics in Lagos University Teaching Hospital, Lagos. West Afr. J. Med., 21: 226-229.

Jarousha, A.M.K.A., I.A. El Qouqa, A.H.N. El Jadba and A.S. Al Afifi, 2008. Acinetobacter baumannii infection in the neonatal intensive care unit. Iranian J. Publ Health, 37: 107-112.

Joly-Guillou, M.L., 2005. Clinical impact and pathogenicity of Acinetobacter. Clin. Microbiol. Infect., 11: 868-873. DOI: 10.1111/j.14690691.2005.01227.x

Joshi, S.G., G.M. Litake, M.G. Satpute, N.V. Telang and V.S. Ghole et al., 2006. Clinical and demographic features of infection caused by Acinetobacter species. Indian J. Med. Sci., 60: 351-360. DOI: 10.4103/0019-5359.27219
Kessaris, A., M. Kravaritt, O. Postolopoulou, D. Bakola and D. Sfiras, 2006. The incidence of infections caused by multi-drug resistant. Acinetobacter Baumannii ICU, 19: 232-236.

Lowman, W., T. Kalk, C.N. Menezes, M.A. John and M.P. Grobusch, 2008. A case of communityacquired Acinetobacter baumannii meningitis-has the threat moved beyond the hospital? J. Med. Microbiol., 57: 676-678. DOI: 10.1099/jmm.0.47781-0

Maragakis, L.L., S.E. Cosgrove, X. Song, D. Kim and P. Rosenbaum et al., 2004. An outbreak of multidrugresistant Acinetobacter baumannii associated with pulsatile lavage wound treatment. JAMA, 292: 3006-3011. DOI: 10.1001/jama.292.24.3006

Michalopoulos, A and M.E. Falagas, 2007. Therapeutic Strategies for Acinetobacter baumannii infections. Eur. Infect. Dis., 125-127.

Munoz-Price, L.S. and R.L. Weinstein, 2008. Current concepts: Acinetobacter infection. N Engl. J. Med., 358: 1271-1281. DOI: 10.1056/NEJMra070741

Oberoi, A., A. Aruna and. M. Lal, 2009. A decade of an underestimated nosocomal pathogen-acinetobacter in a tertiary care hospital in punjab. JK Sci., 11: 2426.

Popescu, G.A., C.G. Liana and A. Streinu-Cercel, 2011. Antimicrobial resistance of acinetobacter baumannii strains isolated in "MATEI BALS" national institute of infectious diseases. Therapeutics, Pharmacol. Clin. Toxicol., 15: 225-229.

Raka, L., S. Kalenc, Z. Boanjak, A. Budimir and S. Katic et al., 2004. Molecular epidemiology of Acinetobacter baumannii in central intensive care unit in kosova teaching hospital. Brazilian J. Infect. Dis., 13: 408-413. DOI: 10.1590/S141386702009000600004

Villegas, M.V. and A.I. Hartstein, 2003. Acinetobacter outbreaks, 1977-2000. Infect. Control Hosp. Epidemiol., 24: 284-295. DOI: 10.1086/502205 